\title{
Afzelia africana Smith ex Persoon dans le Nord-Cameroun. Etat actuel des peuplements et utilisation pastorale
}

\author{
J. Onana ${ }^{1 *}$ J.-L. Devineau ${ }^{2}$
}

\section{Mots-clés}

Afzelia africana - Plante ligneuse Plante fourragère - Plante d'abroutissement - Structure de la population Régénération - Cameroun.

\section{Résumé}

Après un bref rappel de l'importance des ligneux dans les bilans fourragers des systèmes d'élevage en zone semi-aride d'Afrique, une étude de la structure des populations d'Afzelia africana (une des espèces les plus appétées) a été abordée suivant un gradient de la pression pastorale. L'analyse des résultats a permis de distinguer trois groupes structuraux tous caractérisés par une tendance à la régénération des populations. Des itinéraires techniques de gestion ont été proposés en vue de pérenniser cette ressource menacée de disparition.

\section{INTRODUCTION}

Les savanes soudano-sahéliennes fournissent encore la majeure partie du fourrage consommé tant en milieu paysan que dans les centres urbains pour l'alimentation des ruminants domestiques. $\mathrm{Si}$, au cours de la saison des pluies, l'herbe est abondante et de bonne qualité assurant ainsi une relative auto-suffisance alimentaire, au cours de la saison sèche, elle est réduite à l'état de paille sur pied lorsqu'elle n'est pas consumée par les feux de brousse. Pendant cette période, les ressources herbacées seules ne suffisent pas à assurer l'alimentation qualitative des animaux.

Le rôle important des espèces ligneuses dans les bilans fourragers des systèmes d'élevage des zones semi-arides d'Afrique $(7,11,17)$ ainsi que les effets défavorables d'un fort recouvrement et d'une densité élevée sur le développement de la strate herbacée sont depuis longtemps signalés par plusieurs auteurs $(3,12)$. La végétation ligneuse concourt principalement au maintien du potentiel de

\footnotetext{
1. Institut de recherches agricoles pour le développement, Station polyvalente, BP 1073, Garoua, Cameroun

Tél. : +237 7564871 ; fax : +2372272420

2. Antenne IRD, 01 BP 171, Bobo-Dioulasso 01, Burkina Faso

* Auteur pour la correspondance
}

production des parcours. Elle subit actuellement une régression généralisée sous l'effet de la baisse constante des précipitations depuis les années soixante (5), de l'extension des surfaces cultivées (22), des systèmes d'élevage entraînant souvent la surexploitation de cette ressource et d'une demande urbaine en bois en augmentation rapide.

Dans le Nord-Cameroun, qui est limité au sud par la province de l'Adamaoua, en dehors des travaux généraux de phytogéographie (18), de malherbologie (16), des travaux plus spécifiques orientés vers l'utilisation pastorale des savanes ont été menés (20,21, 22, 23) ou sont en cours. Il ressort de ces travaux qu'Afzelia africana est l'une des espèces fourragères les plus utilisées de la région (23), d'où tout l'intérêt qui lui est accordé. La surexploitation de ce fourrage, associée à la rudesse du climat et aux feux de brousse très fréquents dans la région, affecte les possibilités de régénération de l'espèce. Le présent travail qui s'est inscrit dans le cadre général des «Etudes et amélioration des pâturages naturels » de l'Institut de recherches agricoles pour le développement (Irad) du Cameroun a eu pour but d'analyser l'état actuel des peuplements d'Afzelia africana dans la province du Nord-Cameroun en relation avec la pression pastorale. L'objectif final a été de proposer des itinéraires techniques de gestion de cette espèce compatibles avec ses potentialités biologiques. 


\section{MATERIEL ET METHODES}

\section{Distribution géographique et importance d'Afzelia africana dans les systèmes d'élevage du Nord-Cameroun}

Afzelia africana est une légumineuse arborée de la famille des césalpiniacées pouvant atteindre 25-30 (voire 35) m de hauteur et $1 \mathrm{~m}$ de diamètre (1). Les fleurs sont regroupées en panicules à l'extrémité des rameaux de l'année. Son aire s'étend du Sénégal à l'Ouganda $(1,2)$ en passant par la République démocratique du Congo, jusqu'au Soudan (9). Au Cameroun, cette espèce est abondante dans le bassin de la Bénoué. Cette essence est connue localement sous les noms vernaculaires de Gila ou Ndela en Sara, Fao en Toupouri, Ngayodje en Fulfuldé, Tagela en Sara Ngambaye. Les mêmes noms vernaculaires sont signalés pour les régions voisines du Tchad (8).

\section{Milieu de l'étude}

L'étude a été menée dans une zone comprise entre $8-12^{\circ}$ de latitude $\mathrm{N}$ et $12^{\circ} 30^{\prime}-14^{\circ}$ de longitude E. Le climat est de type soudano-sahélien avec deux saisons. Avril est le mois le plus chaud $\left(32{ }^{\circ} \mathrm{C}\right)$. Les principales activités économiques de la région sont l'élevage et l'agriculture. Devant la diversité pédologique rencontrée dans la région, seules les stations sur sols ferrugineux qui couvrent la majeure partie de la zone ont été prospectées. Les relevés ont été réalisés dans le parc de la Bénoué et à Tchéboa (zone cynégétique de l'école de faune de Garoua), Sanguéré-Paul, Laendé Massa, Godé et Ngong.

\section{Estimation de la pression pastorale}

L'élevage dans la zone de l'étude est de type extensif, ce qui expliquait la difficulté à obtenir la charge précise en bétail des pâturages sur lesquels le travail a été effectué. La pression pastorale a été estimée de façon indirecte en utilisant, premièrement, la densité de fèces des ruminants domestiques (DF), aussi bien de l'année que des années antérieures, qui a été divisée selon trois classes (classe $1: \mathrm{DF} \leq 1$ p. 100 ; classe $2: 1<\mathrm{DF} \leq 20$ p. 100 ; classe 3 : DF $>20$ p. 100) ; deuxièmement, trois classes ont également été considérées pour estimer la densité du piétinement (DP) (classe 1 : $\mathrm{DP} \leq 1$ p. 100 ; classe $2: 1<\mathrm{DP} \leq 50$ p. 100 ; classe $3: \mathrm{DP}>50$ p. 100) ; et, troisièmement, les dégâts des animaux sur les plantes fourragères (DV) ont aussi été codés en trois classes [classe 1 pour les stations sur lesquelles la flore fourragère (herbacée comme ligneuse) a présenté très peu de traces de prélèvements ; classe 2 pour celles sur lesquelles ces traces ont porté sur moins de la moitié des plantes présentes ; classe 3 pour les stations sur lesquelles plus de 50 p. 100 des organes de végétaux fourragers à la portée des animaux ont présenté des traces de prélèvement].

Un indice de la pression pastorale apparente (IP) a été obtenu avec la somme de ces trois indices $(\mathrm{IP}=\mathrm{DF}+\mathrm{DP}+\mathrm{DV})$ et a permis de classer les stations en trois catégories : stations non ou faiblement pâturées (IP $\leq 3)$, stations moyennement pâturées $(3<$ IP $\leq 6$ et station surpâturées (IP > 6).

\section{Analyse de l'état actuel des populations}

L'analyse des populations ligneuses peut se réaliser autour des trois éléments suivants (6) :

- la répartition des individus en classes de taille ou de diamètre comme indicateur indirect du niveau d'équilibre des classes d'âge et comme témoin des phases vécues par la population en termes de perturbation ou de régénération ;

- le mauvais état général des peuplements ;
- l'intensité de la régénération, gage du renouvellement à terme de la population.

Vingt relevés de structures de populations ont été réalisés. Ils ont été codés par des caractères alpha-numériques composés de la première lettre du nom de la localité où ils ont été réalisés, soit B pour le parc de la Bénoué, T pour Tchéboa, $\mathrm{S}$ pour Sanguéré-Paul, $M$ pour Laendé Massa, G pour Godé et N pour Ngong. Le numéro accompagnant la lettre indique le rang du relevé dans la série réalisée au cours de la campagne qui portait sur d'autres espèces comme Prosopis africana, Terminalia laxiflora et Daniellia oliveri.

La méthode de recensement utilisée a consisté à mesurer la circonférence du tronc à $80 \mathrm{~cm}$ de 150 pieds, au moins, voisins dans l'espace. Une marque a été faite à la machette sur les individus mesurés pour éviter une répétition. Toutes les plantules présentes dans les relevés ont également été dénombrées afin de mieux appréhender l'état de régénération de la population. La surface du relevé a été notée pour estimer la densité de la population. Toutes les données ont été ramenées à l'hectare afin de comparer les différentes populations par des analyses statistiques.

Dans la présente étude, seuls les individus vivants ont été pris en compte, l'analyse de la mortalité n'ayant pas été faite. Pour mieux comprendre le fonctionnement des populations d'Afzelia africana, des observations complémentaires sur la multiplication végétative de cette espèce ont été entreprises. Elles ont consisté à recenser le nombre de rejets par souche. Seule la circonférence du plus grand a été notée pour matérialiser l'individu. Des observations ont également été faites sur l'enracinement des plantules et des souches de petite circonférence en creusant autour de certaines d'entre elles jusqu'à l'extrémité du système racinaire. Cette opération a permis de déterminer l'origine caulinaire ou racinaire des rejets.

\section{Traitement des données}

Les mesures de circonférences réalisées dans chaque relevé et ensuite ramenées à l'hectare ont été regroupées en huit classes (02, 2-4, 4-8, 8-16, 16-32, 32-64, 64-128, > $128 \mathrm{~cm})$. Chaque classe admet uniquement la borne supérieure.

Pour mettre en évidence les principaux types de distribution des circonférences rencontrés sur l'ensemble des relevés structuraux, une analyse factorielle des correspondances (Afc) (25) a été réalisée sur un tableau de contingence comportant en lignes les classes de circonférences et en colonnes les relevés structuraux. La cohérence des regroupements des relevés obtenus à partir de l'Afc a été vérifiée en comparant l'allure de tous les histogrammes à l'intérieur d'un même groupe structural. Un histogramme moyen a été tracé pour chaque groupe. Les différentes courbes de tendances sur la distribution des individus dans les différentes classes de circonférences ont été comparées aux modèles théoriques élaborés pour des populations présentant une bonne régénération : $\mathrm{Y}=\mathrm{Y}_{0} \mathrm{X}^{-\mathrm{n}}$.

\section{RESULTATS}

\section{Analyses factorielles des correspondances}

Le tableau I donne les caractéristiques de chaque relevé du point de vue de la pression pastorale estimée à partir des densités des fèces et du piétinement, et des dégâts observés sur la flore fourragère.

L'examen des taux d'inertie et des valeurs propres des axes principaux obtenus à partir des analyses factorielles des correspondances (tableau II) montre que la quasi-totalité de l'information contenue dans le tableau de contingence relevés/classes de circonférences est extraite avec les trois premiers axes (respectivement 89,1 p. 100 et 0,639 ). Les deux premiers axes indiquent un taux d'inertie cumulé de 77,1 p. 100, ce qui est déjà en soi intéressant. 


\section{Tableau I}

Estimation de la pression pastorale apparente dans les sites des relevés de structures de populations d'Afzelia africana à partir des densités de fèces et de

piétinement, et des dégâts sur la flore fourragère

\begin{tabular}{lllll} 
Relevé & \multicolumn{2}{c}{ Classe de facteurs } & IP \\
\cline { 2 - 4 } & DF & DP & DV & \\
\hline B6 & 1 & 2 & 2 & 5 \\
B7 & 2 & 2 & 2 & 6 \\
B8 & 1 & 2 & 2 & 5 \\
B9 & 1 & 3 & 2 & 6 \\
B11 & 1 & 2 & 1 & 4 \\
B14 & 1 & 1 & 1 & 3 \\
B15 & 1 & 1 & 1 & 3 \\
G45 & 1 & 1 & 1 & 3 \\
M18 & 1 & 1 & 1 & 3 \\
M19 & 3 & 3 & 2 & 8 \\
N1 & 3 & 3 & 3 & 9 \\
N2 & 3 & 3 & 3 & 9 \\
T24 & 1 & 1 & 1 & 3 \\
T25 & 3 & 3 & 3 & 9 \\
T26 & 1 & 3 & 1 & 5 \\
T30 & 3 & 3 & 2 & 8 \\
T31 & 2 & 3 & 3 & 8 \\
T32 & 2 & 2 & 3 & 7 \\
T34 & 1 & 1 & 1 & 3 \\
S1 & 1 & 1 & 1 & 3 \\
& & & & \\
\hline & & & & \\
\hline
\end{tabular}

DF : densité de fèces. 1 : recouvrement de fèces au sol $\leq 1 \% ; 2: 1 \%<$ recouvrement $\leq 20 \% ; 3$ : recouvrement $>20 \%$

DP : densité de piétinement. 1 : DP $\leq 1 \% ; 2: 1 \%<$ densité $\leq 50 \% ; 3:$ densité $>50 \%$

DV : dégâts sur les végétaux. 1 : dégâts sur la flore fourragère accessible nuls à faibles ; 2 : prélèvements sur moins de $50 \%$ de la flore fourragère accessible ; 3 : prélèvements sur plus de $50 \%$ de la flore fourragère accessible

IP : indice de pression pastorale. $\mathrm{IP}=\mathrm{DF}+\mathrm{DP}+\mathrm{DV}$ (IP $\leq 3$ : faible $; 3<\mathrm{p} \leq 6$ : moyenne ; IP > 6 : forte)

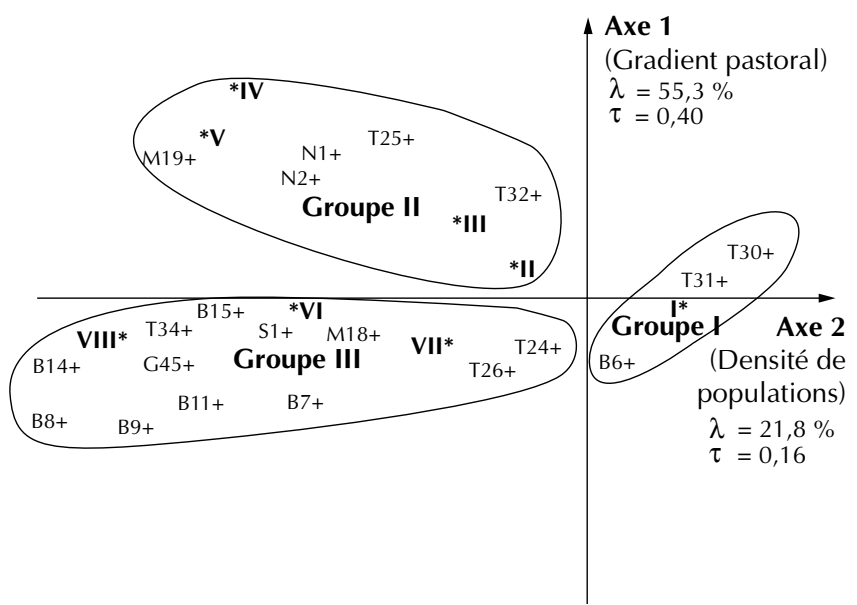

Figure 1 : projection dans le plan des axes 1 et 2 de l'analyse factorielle des correspondances sur la structure des populations d'Afzelia africana Smith ex Persoon des relevés $(+)$ et des classes de circonférences (*). Groupes alpha numériques : la lettre représente la lettre initiale du site, le chiffre le numéro d'ordre du relevé.

\section{Tableau II}

Valeurs propres $(\lambda)$ et taux $d^{\prime}$ inertie $(\tau)$ des axes principaux issus des analyses factorielles des correspondances

\begin{tabular}{lccc} 
Axe & $\begin{array}{c}\text { Valeurs } \\
\text { propres }(\lambda)\end{array}$ & $\begin{array}{c}\text { Taux d'inertie } \\
\tau(\%)\end{array}$ & $\begin{array}{c}\text { Cumul taux } \\
\text { d'inertie } \tau(\%)\end{array}$ \\
\hline 1 & 0,3971 & 55,3 & 55,3 \\
2 & 0,1568 & 21,8 & 77,1 \\
3 & 0,0860 & 12,0 & 89,1 \\
4 & 0,0406 & 5,7 & 94,8
\end{tabular}

Le premier axe de l'Afc (plan des axes 1 et 2) oppose les relevés des stations non ou peu pâturées aux relevés des stations surpâturées et peut dont être interprété comme représentatif d'un gradient pastoral (figure 1). L'axe 2 ordonne les relevés suivant la densité de la population (celle-ci étant beaucoup plus liée à la régénération, donc à l'abondance des individus jeunes). Le troisième axe semble discriminer les relevés suivant leur position géographique ce qui laisse penser à une influence du gradient climatique sur les structures de populations observées. Cette Afc met en évidence trois groupes structuraux (GI, GII, GIII) dont le plus fréquemment rencontré (GIII) éclate en deux sous-groupes dans le plan factoriel des axes 2 et 3 (figures 1 et 2 ) :

\section{Groupe I}

Ce groupe s'est distingué des autres par une forte densité d'individus jeunes (3 500-6 500 pieds.ha ${ }^{-1}$ ). L'histogramme décrivant cette structure a une allure régulièrement descendante vers la gauche (figure 3). C'est le seul groupe structural dont la courbe de tendance tend à s'ajuster au modèle théorique ( $\mathrm{Y}=2$ 986,7 $\left.\mathrm{X}^{-1,4629} ; \mathrm{R}^{2}=0,5172\right)$. C'était à partir de cette structure reflétant un certain état d'équilibre que les autres groupes structuraux ont été déterminés en fonction soit d'une perturbation (permanente ou temporaire), soit de la protection de la régénération. Cette structure

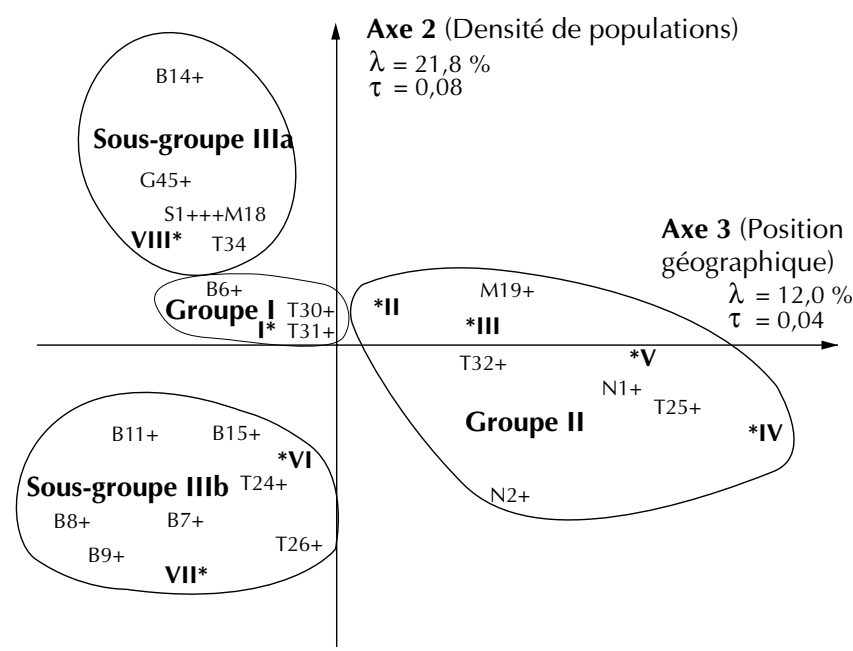

Figure 2 : projection dans le plan des axes 2 et 3 de l'analyse factorielle des correspondances sur la structure des populations d'Afzelia africana Smith ex Persoon des relevés $(+)$ et des classes de circonférences (*). Groupes alpha numériques : la lettre représente la lettre initiale du site, le chiffre le numéro d'ordre du relevé. 
a été uniquement rencontrée dans deux stations surpâturées mais où la densité des individus reproducteurs a été relativement élevée $\left(100\right.$ à 200 pieds.ha $\left.^{-1}\right) ; 10$ p. 100 des relevés effectués ont présenté cette structure.

\section{Groupe II}

Les histogrammes de ce groupe se caractérisent par une structure plurimodale qui s'ajuste par une courbe polynomiale d'ordre 4 à trois modes bien distincts correspondant à trois cohortes de circonférences bien individualisées (figure 3 ) :

- une cohorte constituée des plantules et des rejets de faibles circonférences (classe 1 de régénération) ;

- une cohorte englobant les individus jeunes et moyens des classes de circonférences 2 à 6 ; elle présente une distribution presque normale centrée sur la classe modale 4 ;

- une cohorte qui englobe les individus âgés des classes 7 et 8 .

L'existence de plusieurs cohortes individualisées met en évidence l'alternance des différentes phases de régénération et de perturbation qu'ont connue les populations étudiées. Ces différentes phases pouvaient résulter de pressions zoo-anthropiques anciennes (surpâturage, émondage sévère, activités d'agriculture), suivies d'un abandon ou, tout simplement, d'une alternance des conditions climatiques favorables et défavorables à une bonne régénération de l'espèce. Cette structure a été présente dans les stations surpâturées et accessoirement dans celles qui ont eu des pressions pastorales plus faibles ; 25 p. 100 des relevés effectués ont composé ce groupe structural.

\section{Groupe III}

Ce groupe structural a été le plus représenté dans la zone et a concerné 65 p. 100 des relevés effectués. Il comprenait les relevés dont les populations avaient une structure unimodale en U. Deux sous-groupes, décrits ci-après, ont été distingués (figure 3).

\section{Sous-groupe IIIa}

Ce sous-groupe s'est caractérisé par l'absence d'individus dans les classes de circonférences moyennes. Cette structure a été mise en évidence dans les milieux non ou peu pâturés ayant présenté des densités de populations assez faibles (densité de population $<150$ pieds.ha ${ }^{-1}$ ) qui dénotaient une installation récente de la population ou alors d'un vieillissement de celle-ci (25).

\section{Sous-groupe IIIb}

Les relevés de ce sous-groupe structural ont montré des densités de populations assez élevées et les classes des individus moyens ont été bien représentées. Cette structure a présenté un état de vieillissement des populations avec cependant une bonne régénération.

\section{Régénération}

Tous les histogrammes de structures de populations ont présenté de forts effectifs dans la plus petite classe de circonférences $(<2 \mathrm{~cm})$. Cette abondance de plantules peut être considérée comme un indice appréciable de la régénération. Ces résultats sont en accord avec le pouvoir germinatif observé (73 p. 100) en milieu contrôlé chez cette espèce (20).
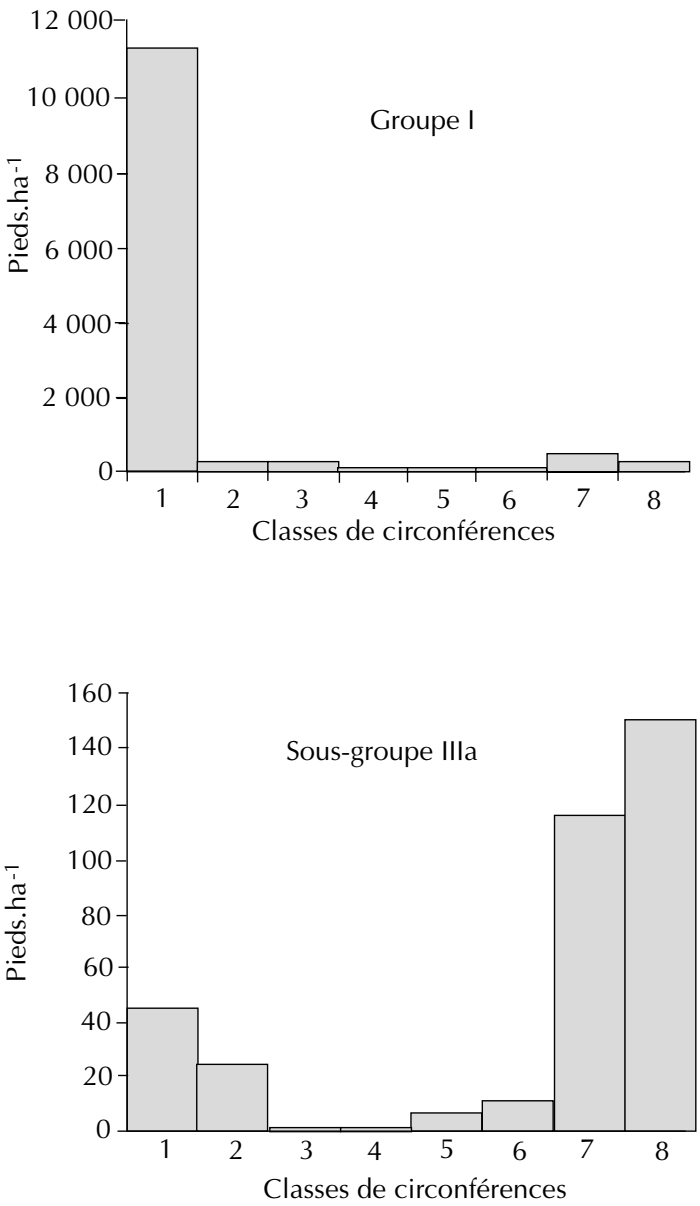
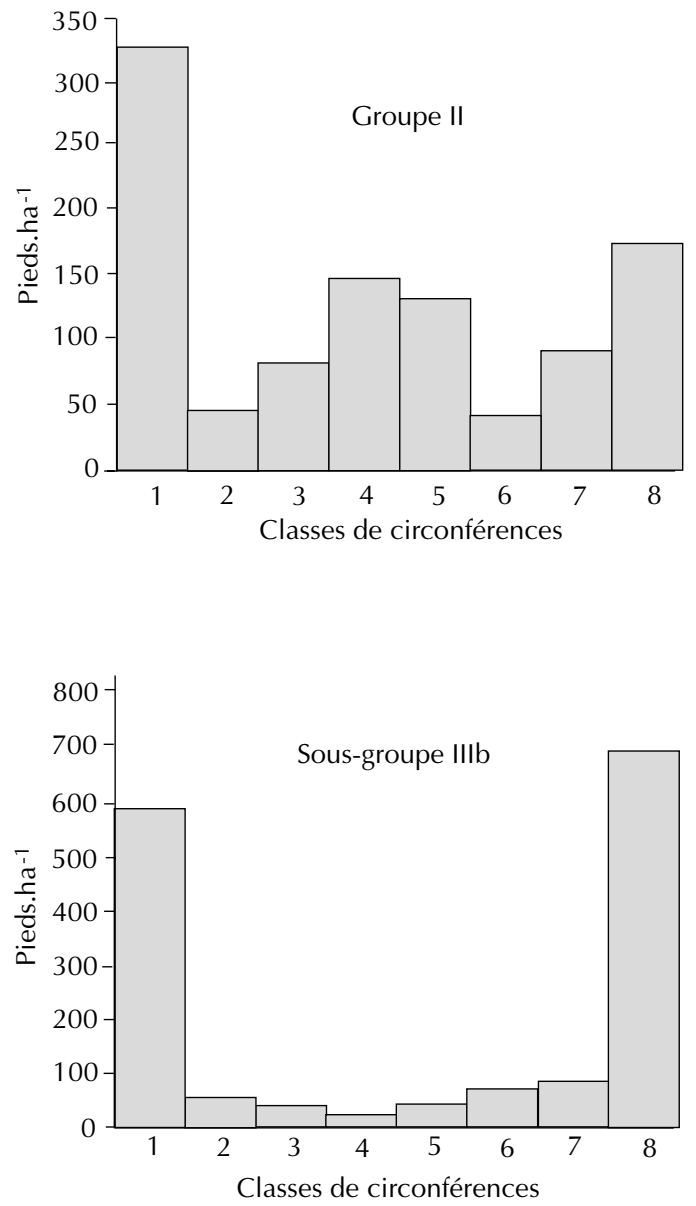

Figure 3 : histogrammes moyens de la distribution des individus par classe de circonférences dans les différents groupes structuraux chez Afzelia africana Smith ex Persoon au Nord-Cameroun. 
Dans les stations peu perturbées, la floraison d'Afzelia africana est généralement importante. Les fruits sont coriaces, très lignifiés, droits et contiennent 5 à 10 graines articulées. Les graines libérées après ouverture des gousses tombent au sol directement sous la couronne de l'arbre à cause de leur poids (une graine pèse $1,55 \mathrm{~g}$ ) (21). A moins qu'elles ne soient entrainées par les eaux de ruissellement, elles germent sur place. La germination est épigée. L'hypocotyle mesure $8,5 \mathrm{~cm}$, l'épicotyle $8 \mathrm{~cm}$ et la radicule $5 \mathrm{~cm}$ en moyenne au stade de deux feuilles végétatives. La croissance initiale en hauteur est très lente en milieu contrôlé (figure 4). En zone soudano-sahélienne, la croissance primaire de l'espèce se caractérise par une phase d'élongation pendant la saison des pluies

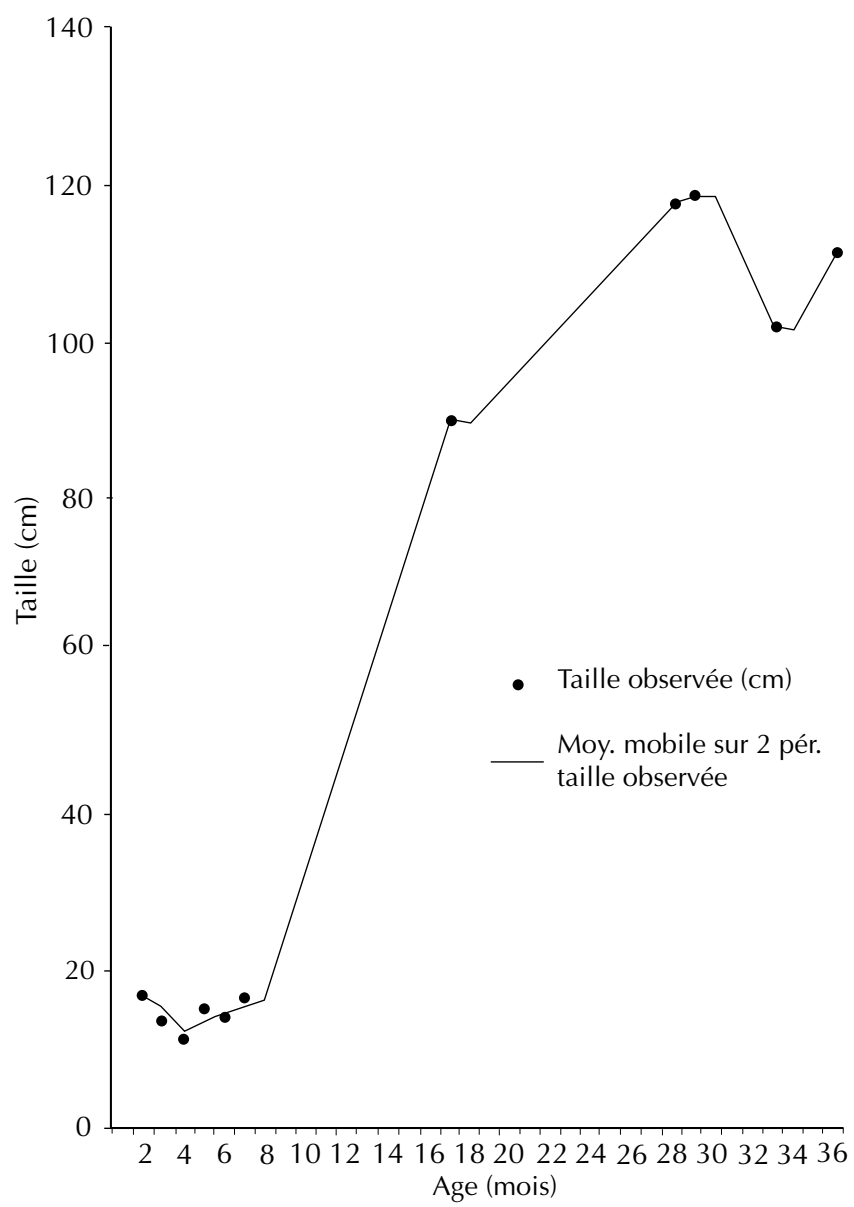

Figure 4 : croissance initiale en hauteur des plants d'Afzelia africana Smith ex Persoon au Nord-Cameroun.

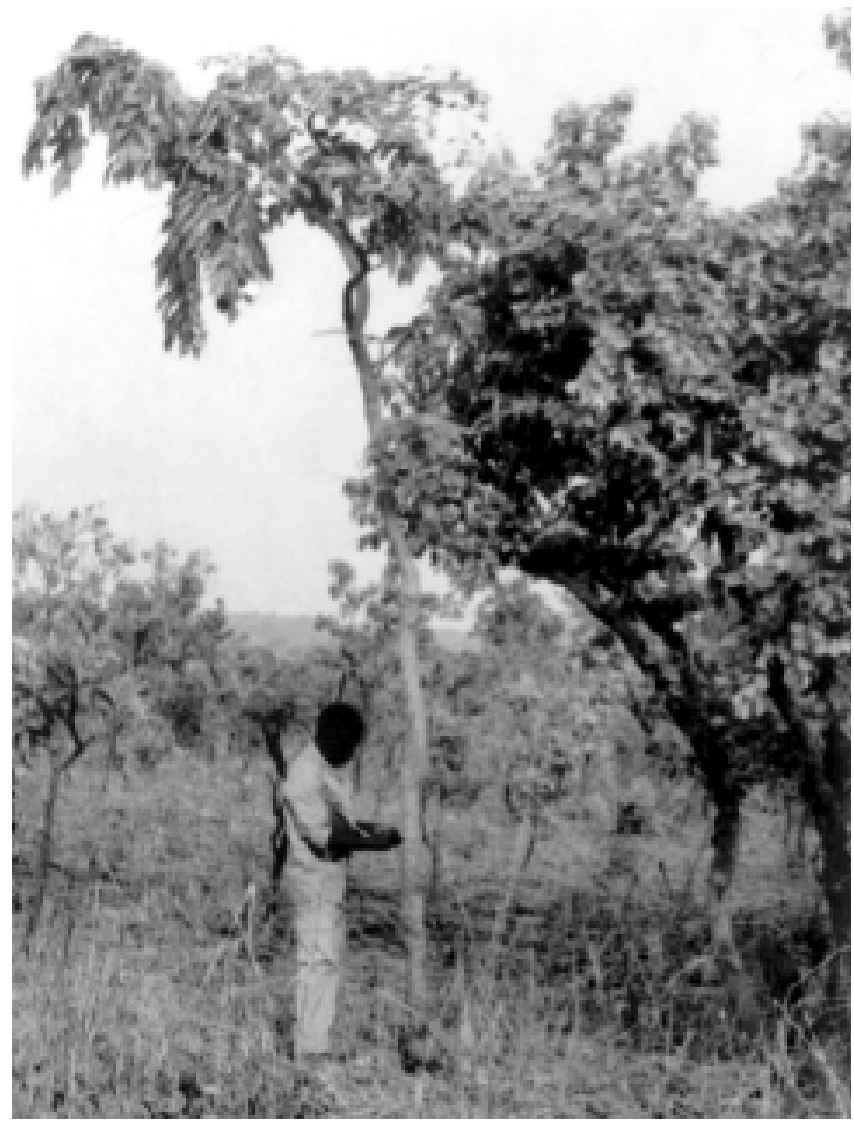

Figure 5 : croissance rythmique chez un jeune plant d'Afzelia africana Smith ex Persoon (cliché de J. Onana, avril 1990, parc national de la Bénoué).

suivie par une phase de repos pendant la saison sèche chaude. Dans certains cas le méristème apical peut même être séché et la croissance en hauteur reprend pendant la nouvelle saison des pluies par un relais issu du bourgeon axillaire immédiat. Ceci se traduit au niveau de l'axe principal par une succession d'unités de croissances (figure 5). La croissance est alors dite rythmique (10).

L'étude entreprise sur le profil racinaire des jeunes Afzelia africana montre que, au cours des premières années, se développe une racine tubérisée, érigée en pivot, sans racines secondaires. Tout traumatisme subi à ce stade par la partie aérienne d'une plantule peut entraîner soit sa mort, soit l'émission de petits rejets à l'occasion d'une pluie. Après plusieurs traumatismes, la souche souterraine peut atteindre des dimensions impressionnantes (tableau III)

\section{Tableau III}

Evolution du nombre de rejets en fonction de la circonférence de la souche mère chez Afzelia africana Smith ex Persoon en stations pâturées

$(\mathrm{N}=14$ relevés, les moyennes portent sur les données ramenées à une surface de $1 \mathrm{ha})$

\begin{tabular}{lccccccc}
$\begin{array}{l}\text { Classe } \\
\text { de circonférences }\end{array}$ & $<\mathbf{2}$ & $\mathbf{2 - 4}$ & $\mathbf{4 - 8}$ & $\mathbf{8 - 1 6}$ & $\mathbf{1 6 - 3 2}$ & $\mathbf{3 2 - 6 4}$ & $\mathbf{6 4 - 1 2 8}$ \\
\hline N1 & $33,0 \pm 6,0$ & $71,0 \pm 14,0$ & $74,0 \pm 22,0$ & $16,0 \pm 2,0$ & $5,0 \pm 2,0$ & $5,0 \pm 1,0$ & $7,0 \pm 5,0$ \\
N2 & $4,0 \pm 0,8$ & $6,3 \pm 2,2$ & $1,5 \pm 0,7$ & $2,0 \pm 0,9$ & $2,1 \pm 1,0$ & $3,0 \pm 2,0$ & $0,4 \pm 0,2$ \\
N3 (\%) & $42,4 \pm 1,7$ & $73,3 \pm 4,2$ & $74,3 \pm 7,4$ & $25,0 \pm 10,0$ & $100,0 \pm 0,0$ & $60,0 \pm 14,3$ & $40,0 \pm 7,8$
\end{tabular}

$\mathrm{N} 1:$ nombre moyen de souches dans un relevé par classe de circonférences

$\mathrm{N} 2$ : nombre moyen de rejets par souche et par classe de circonférences

$\mathrm{N} 3$ : pourcentage de souches présentant des rejets 
et émettre chaque année de petites pousses qui disparaissent rapidement au passage des feux de brousse ou sous les dents des herbivores. Cette faculté à rejeter est importante tant pour la régénération que pour la gestion des arbres à des fins pastorales ou de production de bois de feu $(4,19)$. Le nombre de rejets est variable suivant le diamètre de la souche mère (tableau III). Les souches ayant un diamètre supérieur à $128 \mathrm{~cm}$ n'ont pas été rencontrées lors de relevés.

\section{DISCUSSION}

L'étude dont les résultats viennent d'être présentés a été réalisée dans un milieu où l'élevage est du type extensif, ce qui limite les possibilités de contrôle des pressions d'exploitation sur les ressources naturelles. La zone étant soumise à des contraintes anthropiques fortes, on peut assister à la disparition de nombreuses espèces surexploitées si des mesures de gestion ne sont pas vulgarisées au niveau des utilisateurs. Dans la gestion des ressources fourragères (tant ligneuses qu'herbacées), les principales questions qui se posent sont la protection de la régénération et l'amélioration de la production de biomasse.

Dans la zone de l'étude, Afzelia africana présente de bonnes dispositions naturelles à la régénération. Cependant, la gestion traditionnelle de ce fourrage ligneux qui se fait par émondage annuel ou bisannuel empêche la production de semences. Le surpâturage autour des individus reproducteurs dans une population d'Afzelia peut à long terme entraîner l'épuisement des réserves racinaires au niveau des souches ou des plantules et aboutir à une absence totale de régénération. On obtient alors des populations composées d'une forte proportion d'individus âgés avec très peu (ou pas du tout) d'individus dans les classes moyennes et faibles.

Une bonne application des principes de gestion des espèces fourragères ligneuses déjà acquis $(13,14,15)$ mérite d'être vulgarisée au Nord-Cameroun. Ces principes concernent : (i) la suppression de la pâture sous les porte-graines, (ii) l'émondage différé avec des intervalles de temps favorables à la reconstitution des réserves et (iii) la suppression totale des feux. Des travaux antérieurs $(20,22$, 24) ont montré que leur suppression était favorable au développement des ligneux dans les savanes au Cameroun. La mise en repos temporaire de certains terroirs pastoraux est donc recommandée afin de permettre à certaines générations d'individus d'Afzelia d'échapper à l'action des feux de brousse et de la pâture.

Ces trois conditions indispensables à la régénération semblent difficiles à obtenir dans le contexte social actuel à cause de la gestion communautaire des parcours et des espaces agro-sylvo-pastoraux, d'une pression démographique de plus en plus élevée, du manque d'organisation des éleveurs en coopératives, en résumé de tout ce qui empêche un gel temporaire d'une partie des terrains de parcours. Cependant, l'état actuel des populations d'Afzelia africana dans le Nord-Cameroun permet d'envisager une amélioration des pâturages naturels sur sols ferrugineux en favorisant la régénération de cette espèce dont le rôle dans l'alimentation des ruminants domestiques n'est plus à démontrer.

\section{- CONCLUSION}

Les ligneux fourragers jouent un rôle très important dans l'apport en protéines nécessaire pour un équilibre alimentaire des herbivores domestiques sur pâturage naturel au cours de la saison sèche.

Les résultats qui viennent d'être exposés font le point sur l'état actuel des peuplements d'une des espèces les plus exploitées des parcours. Afzelia africana n'est cependant qu'une composante de l'ensemble pastoral dont la gestion intégrée doit conduire à la pérennisation des ressources. Il s'agit en particulier des groupements herbacés et d'autres espèces fourragères ligneuses exploitées de façon intensive pendant la saison sèche qui posent également des problèmes de gestion.

\section{BIBLIOGRAPHIE}

1. ARBONNIER M., 2002. Arbres, arbustes et lianes des zones sèches d'Afrique de l'Ouest. Paris, France, Muséum national d'histoire naturelle, Montpellier, France, Cirad, $574 \mathrm{p}$

2. AUBREVILLE A., 1970. Flore du Cameroun. Vol. 9. Légumineuses (Césalpiniodées). Paris, France, Muséum national d'histoire naturelle, $339 \mathrm{p}$.

3. AUDRU J., 1977. Les ligneux et subligneux des parcours naturels soudano-guinéens en Côte $d^{\prime}$ Ivoire. Leur importance et les principes d'aménagement de restauration des pâturages. Maisons-Alfort, France, Gerdat-lemvt, 267 p. (Note de synthèse $n^{\circ} 8$ )

4. AUMEERUDOY Y., 1984. Etude de la régénération des arbres par rejets de souches dans la perspective de la production de bois de feu. Mémoire Dea, Ustl, Montpellier, France, 104 p.

5. BOUDET G., 1972. Désertification de I'Afrique tropicale sèche. Andansonia, Série 2, 12 : 205-224.

6. COUTERON P., D'AQUINO P., OUEDRAOGO I.M.O., 1992. Pterocarpus lucens Lepr. dans la région de Banh (nord-ouest du Burkina Faso, Afrique occidentale). Importance pastorale et état actuel des peuplements. Revue Elev. Méd. vét. Pays trop., 45 : 179-190.

7. DAGET P., GODRON M., 1995. Pastoralisme. Troupeaux, espaces et sociétés. Paris, France, Hatier-Aupelf.Uref, p. 206-218.

8. GASTON A., FOTIUS G., 1971. Lexique de noms vernaculaires de plantes du Tchad. Tomes I et II. Maisons-Alfort, France, Gerdat-lemvt, $355 \mathrm{p}$.

9. GEERLING C., 1982. Guide de terrain des ligneux sahéliens et soudano-guinéens. Wageningen, Pays-Bas, Mededelingen Landbouwhogeschool, $340 \mathrm{p}$.

10. HALLE F., OLDEMAN R.A.A., 1970. Essai sur I'architecture et la dynamique de croissance des arbres tropicaux. Paris, France, Masson, $176 \mathrm{p}$.

11. HIERNAUX P., 1980. Inventaire du potentiel fourrager des arbres et arbustes d'une région du Sahel malien. Méthodes et premiers résultats. In : Le Houerou H.N., éd., Les fourrages ligneux en Afrique. Etat actuel des connaissances. Addis Abeba, Ethiopie, Ilca, p. 195-201.

12. HOFFMAN O., 1985. Pratiques pastorales et dynamique du couvert végétal en pays Lobi (nord-est de Côte d'Ivoire). Paris, France, Orstom, $355 \mathrm{p}$.

13. IEMVT-CIRAD, 1989. Les ligneux fourragers et fruitiers en zones tropicale et subtropicale. Paris, France, ministère de la Coopération et du développement, Maisons-Alfort, France, lemvt-Cirad, 8 p. (Fiche technique d'élevage tropical $n^{\circ} 3$ )

14. IEMVT-CIRAD, 1989. Les ligneux fourragers et fruitiers en zones tropicale et subtropicale. Paris, France, ministère de la Coopération et du développement, Maisons-Alfort, France, lemvt-Cirad, 8 p. (Fiche technique d'élevage tropical $\mathrm{n}^{\circ} 6$ )

15. IEMVT-CIRAD, 1989. Les ligneux fourragers et fruitiers en zones tropicale et subtropicale. Paris, France, ministère de la Coopération et du développement, Maisons-Alfort, France, lemvt-Cirad, 8 p. (Fiche technique d'élevage tropical $\left.n^{\circ} 10\right)$

16. LE BOURGEOIS T., 1992. Les mauvaises herbes dans la rotation cotonnière au Cameroun (Afrique) : Amplitude d'habitat et degré d'infestation, phénologie. Thèse Doct., Ustl, Montpellier, France, 204 p. + annexes.

17. LE HOUEROU H.N., 1980. Le rôle des ligneux dans les zones sahéliennes. In : Le Houerou H.N., éd., Les fourrages ligneux en Afrique. Etat actuel des connaissances. Addis Abeba, Ethiopie, Ilca, p. 85-101.

18. LETOUZEY R., 1968. Etude phytogéographique du Cameroun. Paris, France, Lechevalier, $511 \mathrm{p}$.

19. MENAUT J.C., 1971. Etude de quelques peuplements ligneux d'une savane guinéenne de Côte d'Ivoire. Thèse Doct., Université Paris VI, Paris, France, 141 p. 
20. ONANA J., 1990. Pratiques et aménagements sylvo-pastoraux en zone soudano-sahélienne. In : Elevage et potentialités pastorales sahéliennes. Synthèses cartographiques. Maisons-Alfort, France, Ciradlemvt, $23 \mathrm{p}$.

21. ONANA J., 1990. Contribution à l'étude des populations de quelques espèces ligneuses en relation avec la pression pastorale dans la région de Garoua (Nord-Cameroun). Mémoire Dea, Université Paris VI, Paris, France, 42 p. + annexes.

22. ONANA J., 1991. Tendances évolutives de quelques peuplements ligneux pâturés et protégés des feux en zone soudano-sahélienne du Cameroun. In : Séminaire régional sur la gestion des ressources de la biosphère et éducation relative à l'environnement (Projet pilote de Dja), Sangmelima, 6-10 mai 1991. Paris, France, Unesco, p. 305-311.
23. ONANA J., 1995. Les ligneux fourragers du Nord-Cameroun. I. Inventaire et phénologie. Revue Elev. Méd. vét. Pays trop., 48 : 213-219.

24. RIPPSTEIN G., BOUDET G., 1977. Expérimentation sur parcours de savanes tropicales humides. In : colloque Recherches sur l'élevage bovin en zone tropicale humide, Bouaké, Côte d'Ivoire, 18-22 avril 1977. Paris, France, Institut en recherches pour les huiles ou oléagineux, p. 227-243.

25. VOLLE P., 1985. Analyse des données. Paris, France, Economica, $323 \mathrm{p}$.

Accepté le 03/10/2002

\section{Summary}

Onana J., Devineau J.-L. Afzelia africana Smith ex Persoon in North Cameroon. Present State of Populations and Pasture Use

After a brief reminder of the importance of woody plants when assessing feed crops in livestock systems of the semiarid zones of Africa, a study on population structures of Afzelia africana (one of the most grazed species) was carried out based on variations in pasture pressure. The analysis of results distinguished three structure groups all characterized by a tendency of populations to regenerate. Technical methods of management are proposed to help sustain this endangered resource.

Key words: Afzelia africana - Woody plant - Feed crop Browse plant - Population structure - Regeneration - Cameroon.

\section{Resumen}

Onana J., Devineau J.-L. Afzelia africana Smith ex Persoon en el norte de Camerún. Estado actual de las poblaciones y uso pastoril

Después de un corto repaso sobre la importancia de los leñosos en los balances forrajeros de los sistemas de crianza en la zona semi árida de Africa, se aborda un estudio sobre la estructura de las poblaciones de Afzelia africana (una de las especies mas apetecidas), según un gradiente de presión pastoril. El análisis de los resultados permite distinguir tres grupos estructurales, todos caracterizados por una tendencia a la regeneración de las poblaciones. Se proponen itinerarios técnicos de manejo, con el fin de perennizar este recurso, en peligro de desaparición.

Palabras clave: Afzelia africana - Planta lenosa - Planta forrajera - Planta de ramoneo - Estructura de la población - Regeneración - Camerún. 